\title{
Newly Detected Atrial Fibrillation after Acute Stroke: A Narrative Review of Causes and Implications
}

\author{
Youcheng Wang ${ }^{\text {a }}$ Yongsheng Qian $^{\mathrm{a}}$ Daniel Smerin $^{\mathrm{b}}$ Shujuan Zhang ${ }^{\mathrm{a}}$ \\ Qingyan Zhao ${ }^{a}$ Xiaoxing Xiong ${ }^{c}$ \\ ${ }^{a}$ Department of Cardiology, Renmin Hospital of Wuhan University, Cardiovascular Research Institute of Wuhan \\ University, Wuhan City, PR China; ${ }^{b}$ University of Central Florida College of Medicine, Orlando, FL, USA; ${ }^{\mathrm{C}}$ Department \\ of Neurosurgery, Renmin Hospital of Wuhan University, Wuhan City, PR China
}

\section{Keywords}

Acute stroke · Atrial fibrillation - Cardiac autonomic nervous system $\cdot$ Poststroke inflammation

\begin{abstract}
Cardiac arrhythmias occur frequently in patients with acute stroke, with atrial fibrillation (AF) being the most common. Newly detected AF may lead to increased risk of ischemic stroke, which in turn generates stroke recurrence and adverse outcomes. Currently, most studies are focusing on the role of AF in ischemic stroke and attributing cryptogenic ischemic stroke to previously undetected AF. However, in these studies, subjects used to have neither symptoms of palpitation nor evidence of AF. A better understanding of this association will contribute to the management and therapy for patients after clinical decisions regarding stroke patients. Currently, the definition of newly detected AF has not come to an agreement, and the pathophysiology remains incompletely understood, possibly involving complex alterations in both the autonomic network and humoral regulation. Therefore, this review aims to introduce the definition and epidemiology of newly detected AF after stroke with up-
\end{abstract}

\section{KARGER}

(C) 2019 S. Karger AG, Basel

E-Mail karger@karger.com

www.karger.com/crd dated information and elucidate the potential pathophysiology, such as autonomic imbalance, catecholamine surge, poststroke systematic inflammation, and microvesicles and microRNAs.

(c) 2019 S. Karger AG, Basel

\section{Introduction}

Various types of electrocardiographic (ECG) abnormalities and cardiac arrhythmias are commonly observed after an acute cerebrovascular event, including ischemic and hemorrhagic stroke. These patterns of abnormalities vary from abnormal $\mathrm{T}$ waves and QT prolongation to some serious cardiac arrhythmias, such as atrial tachycardia, atrial fibrillation (AF), and even ventricular arrhythmias $[1,2]$. Previous studies have reported a significantly higher frequency of newly detected arrhythmias, primarily AF, in patients with stroke than those without stroke $[2,3]$.

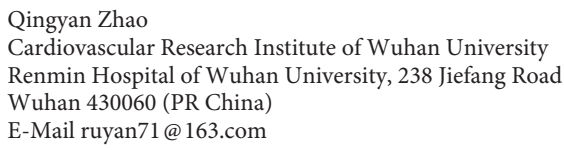


Table 1. Summary of the literature describing the prevalence of poststroke AF

\begin{tabular}{|c|c|c|c|c|c|}
\hline Author & Brain lesion & $n$ & Recording method & $\begin{array}{l}\text { Duration } \\
\text { time }\end{array}$ & $\begin{array}{l}\text { New AF, } \\
\%\end{array}$ \\
\hline Liao et al. [6], 2007 & Acute ischemic stroke & 588 & Holter monitoring & $24-72 \mathrm{~h}$ & 4.6 \\
\hline Lazzaro et al. [7], 2012 & Acute ischemic stroke or TIA & 133 & Holter monitoring & $29.8 \mathrm{~h}$ & 6.0 \\
\hline Thakkar et al. [9], 2014 & Acute ischemic stroke or TIA & 52 & Holter monitoring & $24 \mathrm{~h}$ & 5.8 \\
\hline Grond et al. [8], 2013 & Acute ischemic stroke & 1,135 & Holter monitoring & $72 \mathrm{~h}$ & 4.3 \\
\hline Sanna et al. [11], 2014 & $\begin{array}{l}\text { Cryptogenic ischemic stroke } \\
\text { or TIA }\end{array}$ & $\begin{array}{l}221 \\
220\end{array}$ & $\begin{array}{l}\text { ICM } \\
\text { Conventional follow-up }\end{array}$ & 12 months & $\begin{array}{r}12.4 \\
2.0\end{array}$ \\
\hline Tayal et al. [17], 2008 & $\begin{array}{l}\text { Cryptogenic ischemic stroke } \\
\text { or TIA }\end{array}$ & 56 & MCOT & 21 days & 23.0 \\
\hline Bhatt et al. [13], 2011 & Ischemic stroke or TIA & 62 & MCOT & 28 days & 24.0 \\
\hline Favilla et al. [12], 2015 & $\begin{array}{l}\text { Cryptogenic ischemic stroke } \\
\text { or TIA }\end{array}$ & 227 & MCOT & 28 days & 14.0 \\
\hline Maramattom et al. [21], 2006 & $\mathrm{ICH}$ & 110 & Continuous ECG monitoring & & 12.0 \\
\hline van Bree et al. [20], 2010 & $\mathrm{ICH}$ & 31 & Admission ECG & & 10.0 \\
\hline Frontera et al. [22], 2008 & $\mathrm{SAH}$ & 580 & Continuous ECG monitoring & & 3.2 \\
\hline
\end{tabular}

ECG, electrocardiographic; AF, atrial fibrillation; SAH, subarachnoid hemorrhage; ICH; intracerebral hemorrhage; TIA, transient ischemic attack.

It is well-recognized that AF is the most common sustained cardiac arrhythmia and known as a key risk factor for ischemic stroke, increasing its risk by 5 - to 6 -fold independent of other risk factors [4]. Nevertheless, new AF can also be detected in patients after acute stroke without previous evidence of cardiac arrhythmias. A better understanding of this association will contribute to the management and therapy for patients after clinical decisions regarding stroke patients, such as how to choose an appropriate cardiac recording strategy, and whether to use oral anticoagulant therapy for stroke recurrence prevention.

\section{Prevalence of Newly Detected AF after Stroke}

AF detected after stroke is so complex that no currently used term fits adequately. We propose the term newly detected AF for AF detected by any ECG monitoring technology in patients (no previous past or recent history of AF) after stroke onset, which may consist of both preexisting undetected AF prior to stroke and poststroke AF. Over $70 \%$ of cases have been diagnosed within the first 3 days after admission [5].
The incidence of new AF reported in studies varies from choices of cardiac monitoring device, interval between the start of monitoring and stroke onset, duration of cardiac monitoring, and different types of stroke, which is the most appropriate monitoring method is still up for debate. The literature describing the prevalence of poststroke AF is summarized in Table 1. It is observed that continuous monitoring methods could identify more AF than a routine Holter monitoring after acute stroke [6-14].

Given the paroxysmal characteristic of new AF, current guidelines recommend an ECG monitoring for 24 or more hours to exclude AF in patients with an acute ischemic stroke. However, the most effective and appropriate period of monitoring has not come to an agreement. It has been confirmed that a longer duration of monitoring substantially leads to increased detection of AF after ischemic events $[15,16]$. In an analysis including a total of 8,715 patients from 31 studies, new AF was detected in $5.1 \%$ of patients with monitoring lasting $<72 \mathrm{~h}$, whereas the detection increased to $15 \%$ in studies with monitoring lasting more than 7 days [15].

Time between stroke onset and the start of monitoring should also be taken into consideration. For example, it 
has been reported that initiation of MCOT 21 days after acute stroke yielded a $23 \%$ prevalence of new AF [17]. When intervals extended up to 33 and 77 days after the index event, a 17 and $16 \%$ prevalence of new AF was yielded, respectively $[10,14]$, demonstrating increased detection with earlier monitoring.

However, most of the above mentioned studies emphasized the role of previously undetected AF in acute ischemic stroke and failed to recognize that AF can also be the outcome of acute stroke, which is termed poststroke AF. It has been reported that most cases of poststroke AF could be detected within the first 3 days after admission and disappeared after a few days. The duration and potential recurrence of poststroke AF ranged from 3 to 77 days, 23 days on average [18]. Therefore, we believe that the earlier the episode of AF occurs after stroke, the more evident that $\mathrm{AF}$ will be a consequence of the stroke and not the cause of it.

Additionally, this hypothesis may be partly supported by new AF in patients with hemorrhagic stroke. The prevalence of new AF is less common in patients with hemorrhagic stroke, including intracerebral hemorrhage ( $\mathrm{ICH}$ ) and subarachnoid hemorrhage (SAH), than with ischemic stroke [19-21]. In a cohort of 110 patients with no history of heart disease or ECG abnormalities, ECG abnormalities were identified in $64 \%$ after ICH, in which AF or flutter was $12 \%$ [21]. There are more literature reporting on ECG abnormalities in patients with SAH compared to $\mathrm{ICH}$, and $\mathrm{SAH}$ seems to produce a more pronounced arrhythmogenic risk and clinical outcome [22]. But few studies specifically describe the prevalence of AF in patients after hemorrhagic stroke.

\section{Pathophysiology}

\section{Autonomic Nervous System}

ANS and AF

A number of animal and clinical studies have demonstrated that imbalance of the cardiac autonomic nervous system (ANS) plays a key role in AF occurrence and maintenance, and parasympathetic and sympathetic overactivity can both increase the vulnerability of AF. Early research found that vagal nerve stimulation may induce AF in dogs [23]. The effect of the vagal nerve on the atrium is mainly caused by the release of acetylcholine in the peripheral nerve, which activates acetylcholine potassium channel current on the myocardial membrane, resulting in shorter action potential duration (APD) and atrial effective refractory period (AERP), and a larger dis- persion of AERP [24]. Later, however, a series of studies showed that low-level vagal nerve stimulation can prevent atrial structural remodeling (atrial enlargement and atrial fibrosis) and electrical remodeling (AERP shortening and dispersion of AERP increasing), which is considered as one of the most important mechanisms of genesis and progression of AF, thereby inhibiting AF occurrence $[25,26]$.

In addition to parasympathetic nerve, the role of sympathetic nerve seems to be more significant in the development of AF. It has been demonstrated that the increased distribution of sympathetic nerve in atrium is closely associated with AF inducibility and duration. Therefore, methods trying to attenuate sympathetic activity, such as stellate ganglion ablation and renal sympathetic denervation, can obviously inhibit AF inducibility $[27,28]$.

The principle sympathetic neurotransmitter norepinephrine interacts with $\beta$-adrenergic receptors on cardiomyocytes to execute adrenergic action, which provides a positive effect to $\mathrm{Ca}^{2+}$-dependent cardiac function. However, the enhanced $\mathrm{Ca}^{2+}$ handling produced by $\beta$-adrenergic activation also promotes an ectopic activity via several mechanisms, such as enhanced automaticity, early afterdepolarizations, and delayed afterdepolarizations.

\section{Imbalance of ANS after Acute Stroke}

Clinical and experimental research has shown that autonomic dysfunction is commonly observed after acute stroke, which is generally evidenced by the loss of physiological regulation on heart rate and blood pressure. This abnormality further leads to reduced heart rate variability (HRV) and impaired baroreceptor reflex sensitivity (BRS) and increased catecholamine and cortisol levels [29]. The high-frequent (HF) component of HRV is generally representative of parasympathetic activity, and the low-frequent (LF) component is thought to be the combined sympathetic and vagal response to arterial baroreceptors. Roughly, a relatively greater parasympathetic activity is reflected by a low LF/HF ratio, while a higher sympathetic activity is reflected by a high ratio [30]. In a recent clinical study, HRV abnormalities were found in almost all patients with acute stroke, of whom $60 \%$ had an increased LF/HF ratio, suggesting a higher sympathetic activity [31].

The baroreceptor reflex can stabilize the heart rate and blood pressure when our body position changes. Impaired BRS was reported in patients with acute and subacute stroke [32]. Both reduced HRV and impaired BRS seem to 


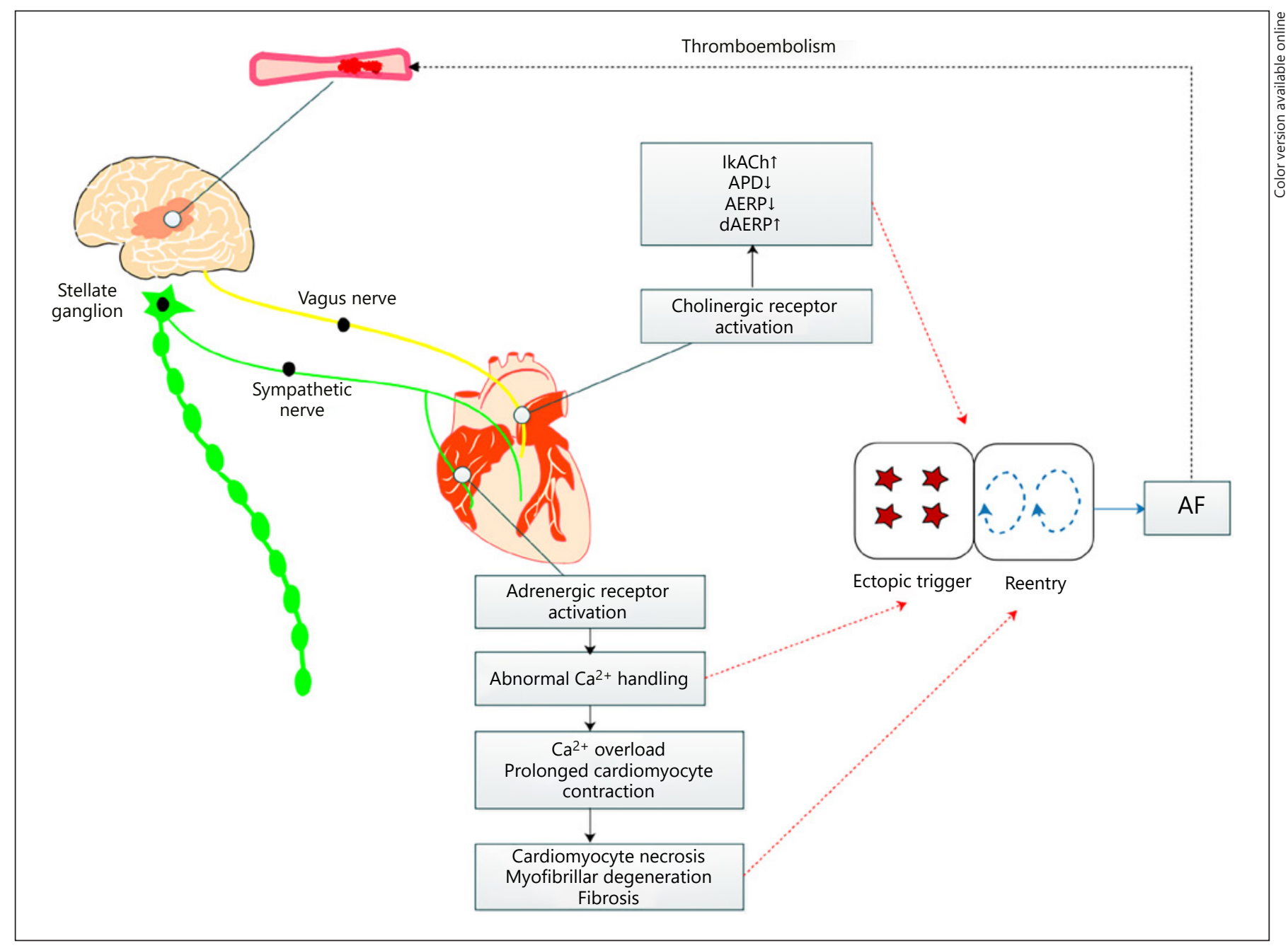

Fig. 1. Neural mechanism of poststroke AF. An acute stroke leads to autonomic imbalance, either parasympathetic overactivity or sympathetic overactivity. The excessive parasympathetic discharge results in a shorter APD and AERP, and a larger dAERP, by activating IkACh, producing the spatial heterogeneity and reentry in atrium. Sympathetic hyperactivity after stroke leads to excessive release of catecholamines, which activates $\beta$-adrenergic receptors

be associated with poorer prognoses and long-term mortality in patients with acute ischemic stroke $[32,33]$.

Taken together, an imbalance of ANS after acute stroke, mainly sympathetic overactivation, may underlie the pathophysiology of AF occurrence. Autonomic imbalance may lead to atrial structural and electrical remodeling, increasing AF inducibility directly or indirectly. The neural mechanism of poststroke AF is described as Figure 1. Notably, poststroke autonomic imbalance might contribute not only to AF but also to other fatal and nonfatal arrhythmias and even myocardial injury [34].

Newly Detected AF after Acute Stroke on myocardial cells. Overactivation of $\beta$-adrenergic receptors can lead to abnormal cardiomyocytes $\mathrm{Ca}^{2+}$ handling, promoting an ectopic activity. Additionally, adrenergic activation can also lead to structural remodeling such as cell necrosis, myofibrillar degeneration, and fibrosis. AF, atrial fibrillation; APD, action potential duration; AERP, atrial effective refractory period; dAERP, dispersion of AERP; IkACh, acetylcholine ACh potassium channel current.

\section{Lesion Location on Cardiac Arrhythmia}

There possibly exists a link between lesion location in the brain and the occurrence of severe cardiac arrhythmias, with the most important area being the insular cortex. The insular cortex and the subinsular white matter, which has blood supplied by the middle cerebral artery, serve as a crucial role in the central regulation of the autonomic network [29]. Damage of the insular cortex was independently associated with poststroke AF, with patients with insular cortex damage showing a 7-fold higher risk of AF than patients without insular involvement [35]. 
Interestingly, the role of the left and right hemisphere in regulation of autonomic nerve function may not be symmetrical. An early study reported that the heart rate decreased in patients with right-hemispheric inactivation by right intracarotid amobartital injection, whereas the increased heart rate was observed after left-hemispheric inactivation by left intracarotid amobartital injection [36], suggesting the right hemisphere primarily controls sympathetic activity, whereas parasympathetic activity is mainly controlled by the left. In a clinical research, including 103 patients with acute ischemic stroke, decreased HRV and frequent complex arrhythmias were more pronounced in subjects with right insular cortex infarction [37], which further suggested an ischemic lesion in the right insular cortex could reduce sympathetic tone and result in parasympathetic overactivity.

However, not all data support this hypothesis. Several clinical studies have suggested that ischemic stroke involving the right insular cortex might produce a much more excessive pathological discharge of the sympathetic nervous system than other regions of brain $[38,39]$. The conflicting evidence indicates that the understanding of location for autonomic control centers may be oversimplified.

Besides the insular cortex, other parts of the cerebrum such as the anterior cingulate cortex, amygdala, parabrachial nucleus, hypothalamus, and periaqueductal gray matter are also involved in the central control of ANS and modulation of cardiac function [40]. Nevertheless, the connection between lesion location in the cerebrum and arrhythmia occurrence, especially AF, has been not clarified.

\section{Catecholamine Surge and Myocardial Damage after Acute Stroke}

Accumulated evidence has suggested both ischemic and hemorrhagic stroke can lead to myocardial damage, even in the absence of coronary heart disease. Elevated levels of TnI in plasma have been reported in up to $34 \%$ of patients with acute ischemic stroke and shown to be dependent on new-onset $\mathrm{AF}$ after ischemic stroke, which might serve as a predictor for the risk of new AF in poststroke patients [41, 42].

The catecholamine surge hypothesis is possibly the most widely accepted mechanism linking myocardial damage to acute stroke. Released from the adrenal medulla and the sympathetic nerve endings, the secretion of catecholamines is mainly regulated by the activity of ANS. Several early studies have reported an elevated cat- echolamine concentration in plasma after acute cerebrovascular events, accompanied by an increased incidence of arrhythmias. Sympathetic hyperactivity after stroke leads to excessive release of catecholamines. Catecholamines can enhance the activity of L-type $\mathrm{Ca}^{2+}$ channel and elevate the concentrations of $\mathrm{Ca}^{2+}$ in cytoplasm by activating $\beta$-adrenergic receptors on myocardial cells, thereby strengthening myocardial contractility. However, overactivation of $\beta$-adrenergic receptors can lead to intracellular $\mathrm{Ca}^{2+}$ overload and the muscle's failure to relax, resulting in metabolic imbalance and cell death [43]. In addition to myocardial necrosis, cardiac hypertrophy, myofibrillar degeneration, and fibrosis have also been reported in patients after stroke [44]. All of these may contribute to structural remodeling and abnormal conduction in the heart, which possibly serves as the substrate for AF occurrence and maintenance (Fig. 1).

\section{Inflammation}

Inflammation after Acute Stroke

Poststroke inflammation is an essential factor in stroke progression and other secondary complications such as brain edema, pathogen infections, and cardiovascular events. In the acute phase of stroke, cerebral injury leads to local inflammatory responses including microgliosis and astrogliosis, resulting in massive secretion of cytokines and chemokines [45], which occurs together with endothelial cell damage [46]. Increased blood brain barrier (BBB) permeability, as a consequence of damaged endothelial cells, facilitates the infiltration of peripheral leukocytes and macrophages into cerebral tissue [47]. Necrotic cells from the ischemic lesion area release damage-associated molecular pattern molecules (DAMPs), such as high mobility group box 1 (HMGB1), heat shock proteins, and Peroxiredoxin (Prx) family proteins, for initial induction of the inflammatory response $[48,49]$.

HMGB1 can not only act the role of cytokine but also enhance the binding of other cytokines to Toll-like receptors (TLRs), especially TLR2 and TLR4, thus mediating inflammatory reaction. In addition to TLRs, HMGB1RAGE-mediated pathway is also responsible for the activation of poststroke inflammation [49]. Prx family proteins are another key DAMP. When released extracellularly from necrotic cerebral cells, Prx proteins act as a neurotoxic danger signal initiating systematic inflammation by binding to TLR2 and TLR4 [48]. A recent study indicated that Prx proteins released extracellularly $12 \mathrm{~h}$ after stroke onset, delayed but existing longer than HMGB1 [48]. 
These DAMPs can primarily stimulate the migration and activation of macrophages via TLR2 and TLR4, which are originally responsible for sweeping away cell debris and helping restore neuronal functions. Nevertheless, once excessively activated, they can produce an adverse effect on neurons and endothelial cells and disrupt neurogenesis [47]. Activated microglia and macrophages polarize into the proinflammatory phenotype M1 and release proinflammatory cytokines [50]. As previously mentioned, increased BBB permeability, which may be further exacerbated by increased inflammatory cytokines, also facilitates these proinflammatory molecules to pass through the damaged $\mathrm{BBB}$ into the peripheral circulation, thereby inducing systematic inflammation.

Except for DAMPs, brain-derived antigens released from injured brain cell are also involved in the poststroke inflammatory response, such as glial fibrillary acidic protein, S100, and myelin basic protein [51]. After stroke, lymphocytes infiltrate the ischemic area for contact with brain-derived antigens from the peripheral immune system [52]. Meanwhile, the serum levels of brain-derived antigens markedly increased in patients after stroke, which might allow lymphocytes to encounter these antigens in peripheral lymphoid organs, inducing systematic inflammation [53].

A recent study has documented that the infiltration of neutrophils, lymphocytes, and macrophages was much higher in the myocardium of patients who died after SAH, suggesting a link between stroke and cardiac local inflammation [54]. However, the relationship between ischemic stroke and cardiac local inflammation has not been clearly reported.

AF Induced by Inflammation

AF may be one of the major secondary complications of systematic inflammation after stroke. It has been demonstrated that the presence of inflammation either in the myocardium or peripheral circulation is associated with AF occurrence and recurrence in postoperative AF [55]. A series of studies that examined interference with intrinsic and extrinsic cardiac autonomic nerves further support the intimate relationship between the levels of inflammatory factors and AF induction [28, 56-58].

Inflammatory mediators gradually lead to the alteration of atrial electrophysiology and structural substrates via different signal pathways, thereby resulting in the increased AF vulnerability. The detailed molecular pathophysiological mechanisms of electrical and structural remodeling in $\mathrm{AF}$ [59] and the association between inflammation and AF [55] have been previously reviewed respectively.

Newly Detected AF after Acute Stroke
Different inflammatory cytokines have distinct influences on the function of ion channels and the change of structural substrates. For example, mice overexpressing tumor necrosis factor (TNF)- $\alpha$ in the heart had prolonged APD, decreased contractile function, and abnormal systolic and diastolic $\mathrm{Ca}^{2+}$ handling compared with a control group [60]. In addition to electrical remodeling, TNF- $\alpha$ can also activate myofibroblasts and increases the secretion of matrix metalloproteinase 2 in mice through the transforming growth factor- $\beta$ signal pathway, which may contribute to atrial fibrosis and AF development [61].

Interleukin (IL)-6 is a pleiotropic cytokine acting as a mediator for both proinflammatory responses and cytoprotective functions. It physiologically promotes the synthesis of multiple inflammatory factors, such as TNF- $\alpha, C$-reactive protein, fibrinogen, and IL-1 $\beta$ [62]. Additionally, IL- 6 promotes the expression of ICAM-1 and the activation of matrix metalloproteinases in cardiocytes, thereby inducing cardiomyocyte apoptosis and fibrosis.

Platelet-derived growth factor (PDGF), mainly produced by myofibroblasts and mast cells, is another inflammatory cytokine that has influence on both electrical and structural alterations in cardiomyocytes. It has been reported that PDGF-A promoted cell proliferation and collagen expression in cardiac fibroblasts in mice, contributing to atrial fibrosis and increased AF susceptibility [63]. Administration of PDGF has also been demonstrated to result in a significant reduction in peak L-type $\mathrm{Ca}^{2+}$ current density and $\mathrm{Ca}^{2+}$ transients in atrial myocytes, as well as APD shortening, which might contribute to atrial electrical remodeling [64].

In summary, after stroke, increased BBB permeability facilitates multiple DAMPs and inflammatory factors to infiltrate into the peripheral circulation, thereby inducing systemic inflammation. Meanwhile, these DAMPs can not only interact with immune cells but also directly activate the TLRs on the myocardial membrane, inducing the synthesis and release of inflammatory factors. Under the different effects of inflammatory factors, irreversible electrophysiological changes and structural remodeling gradually occur in atrium, promoting the occurrence and maintenance of AF. The inflammatory mechanism of poststroke AF is described as in Figure 2.

\section{Microvesicles and MicroRNAs in Poststroke AF}

Microvesicles are small membrane-enclosed vesicles produced by the outward budding and exocytosis of the plasma membrane into the extracellular space by several cell types. They mediate the interconnections among cells 


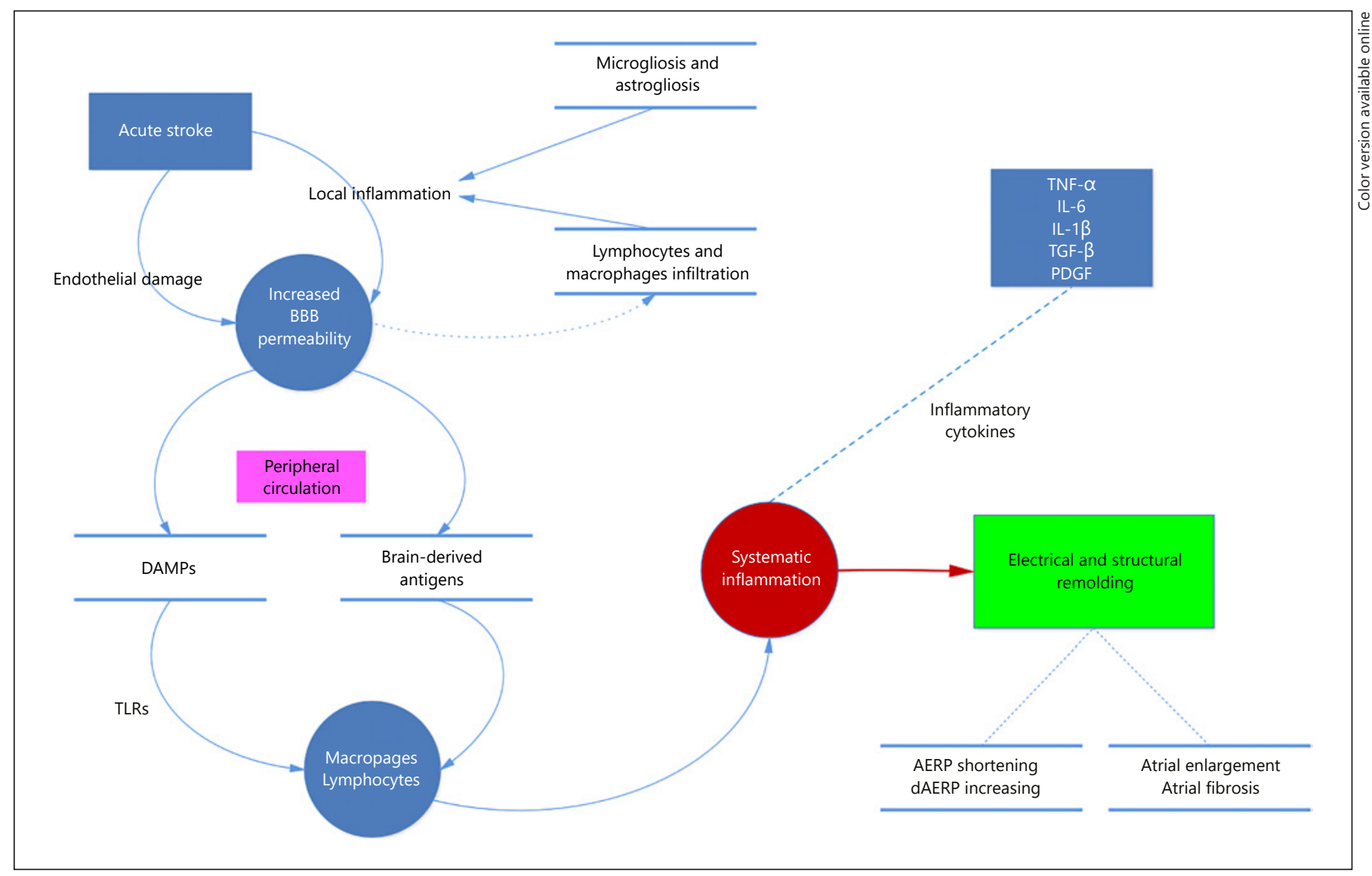

Fig. 2. Inflammatory mechanism of poststroke AF. DAMPs and antigens release from lesion areas after acute stroke, which promotes the filtration of macrophages and lymphocytes and the releasing of proinflammatory cytokines. Increased BBB permeability, which may be further exacerbated by increased inflammatory cytokines, facilitates these proinflammatory molecules to pass through the damaged $\mathrm{BBB}$ into the peripheral circulation, thereby inducing systematic inflammation. The above pathological pro-

to participate in different physiological or pathological processes such as inflammation, coagulation, and tumor progression [65]. A number of studies indicate that microvesicles may play a crucial role in secondary complications after ischemic and hemorrhagic stroke. It has been shown that the circulating levels of microvesicles are markedly elevated in patients with ischemic stroke, ICH, and $\mathrm{SAH}$, and are consistent with a poorer clinical outcome [66-68]. A recent study has shown that the serum levels of microvesicles significantly increased in patients with persistent $\mathrm{AF}$, which might mediate inflammation and platelet activation [69]. Therefore, we hypothesize that microvesicles may also serve as a mediator in poststroke AF. cesses ultimately contribute to the atrial electrical and structural remodeling, which possibly serves as the substrate for the occurrence and maintenance of AF. TNF- $\alpha$, tumor necrosis factor- $\alpha$; IL-6, interleukin-6; IL-1 $\beta$, interleukin- $1 \beta$; TGF- $\beta$, transforming growth factor- $\beta$; PDGF, platelet-derived growth factor; AERP, atrial effective refractory period; dAERP, dispersion of AERP; DAMPs, damaged associated molecular patterns; BBB, blood brain barrier.

MicroRNAs (miRNAs) are highly conserved noncoding RNAs that are abundantly contained in microvesicles and regulate gene expression at either the transcriptional or posttranscriptional level. Recent studies have revealed a pivotal role of miRNAs in the initiation and progress of cardiovascular disease, including AF. The role of miRNAs in the pathophysiology, diagnosis, and prognosis for AF has been exclusively reviewed [70]. Up until now, several miRNAs in atrial tissue, such as miR-1, miR-21, miR-26, miR-29, miR-30a/b, miR-31, miR-328, and miR-208a/b, have been reported to participate in atrial electrical and structural remodeling [70]. Interestingly, some of them like miR-21, miR-29, and miR-30a are also found to be upregulated in plasma samples of patients with acute 


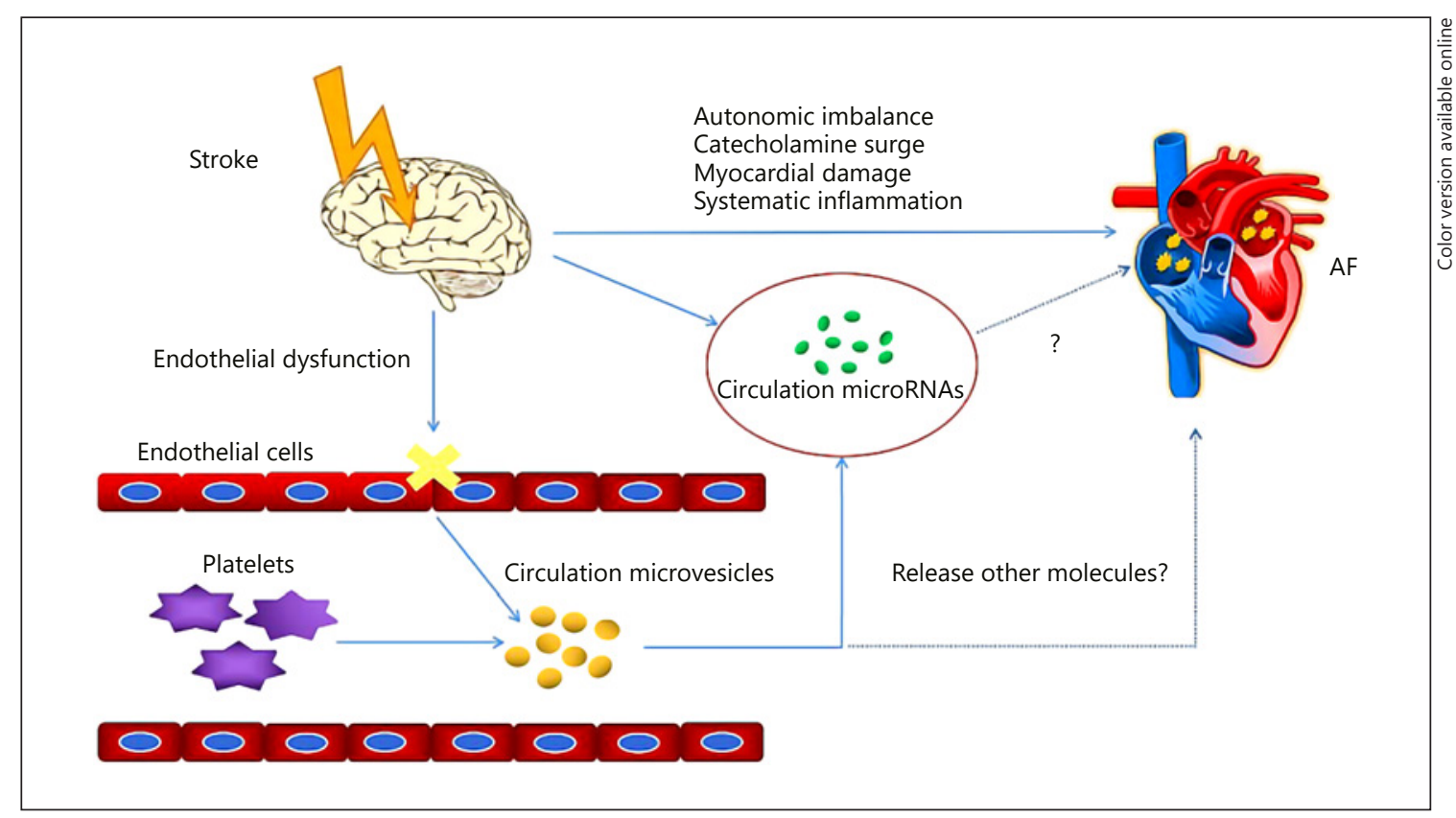

Fig. 3. The possible role of microvesicles and miRNAs in poststroke AF. The levels of circulation microvesicles, mainly produced by endothelial cells and platelets, markedly increased after an acute stroke. Microvesicles may serve as a mediator in inflammation and poststroke AF by releasing miRNAs or other mole-

stroke [71]. miR-126 plays a pivotal role in vasculogenesis and is considered a prognostic marker for vascular injury and endothelial dysfunction. In patients with acute ischemic stroke, the level of circulating miR-126 has been reported to be markedly downregulated [72]. The decreased serum level of miR-126 is thought to be highly associated with cardiovascular diseases such as AF, heart failure, and myocardial infarction [73], which supports the hypothesis that miR-126 may serve as a key mediator in poststroke cardiac complications. The role of microvesicles and miRNAs in the development of poststroke AF remains to be further studied. The possible role of microvesicles and miRNAs in poststroke AF is summarized in Figure 3.

\section{Conclusion and Future Perspective}

New AF after a cerebrovascular event is a frequent finding, and its clinical impact is so high that new therapeutic strategies have been proposed in patients who may have a paroxysmal nondetected arrhythmia. For this reason, the understanding of the pathophysiological mechanisms of AF after stroke is crucial and could provide new targets for future therapies in patients with stroke.

Newly Detected AF after Acute Stroke cules. Some miRNAs may participate in atrial electrical and structural remodeling in $\mathrm{AF}$ and their concentrations in plasma change after an acute stroke. The specific association needs to be further clarified. AF, atrial fibrillation.

Poststroke pathophysiology is complex and possibly involves alterations in both the autonomic network and humoral regulation. Autonomic imbalance, catecholamine surge, neurogenic cardiac damage, and systematic inflammation after acute stroke can lead to atrial electrical remodeling and structural remodeling via different pathways, thereby facilitating the occurrence and maintenance of AF. Interestingly, according to a recent clinical study, the risk of ischemic recurrence in patients with poststroke AF seems to be lower compared to patients with AF diagnosed prior to stroke, suggesting different pathophysiology and cardiac features from general AF [74].

In addition, future researches are needed to clarify the association between the lesion area and arrhythmia occurrence, which may contribute to the treatment and management of patients with acute stroke.

Microvesicles and miRNAs are thought to play a pivotal role in the initiation and maintenance of AF and other cardiac complications after acute stroke. However, their underlying mechanisms remain unclear. We hypothesize that microvesicles and miRNAs may serve as a new predictive biomarker or therapeutic target for poststroke AF and other cardiac complications. 


\section{Acknowledgement}

This work was supported by the National Natural Science Foundation of China (No. 81571147 to X. Xiong and No. 81670303 to Q.Y. Zhao).

\section{Disclosure Statement}

The authors have no conflicts of interest.

\section{References}

1 Norris JW, Froggatt GM, Hachinski VC. Cardiac arrhythmias in acute stroke. Stroke. 1978 Jul-Aug;9(4):392-6.

2 Goldstein DS. The electrocardiogram in stroke: relationship to pathophysiological type and comparison with prior tracings. Stroke. 1979 May-Jun;10(3):253-9.

3 Kallmünzer B, Breuer L, Kahl N, Bobinger T, Raaz-Schrauder D, Huttner HB, et al. Serious cardiac arrhythmias after stroke: incidence, time course, and predictors-a systematic, prospective analysis. Stroke. 2012 Nov; 43(11):2892-7.

4 Romero JR, Wolf PA. Epidemiology of Stroke: Legacy of the Framingham Heart Study. Glob Heart. 2013 Mar;8(1):67-75.

5 Sposato LA, Klein FR, Jáuregui A, Ferrúa M, Klin P, Zamora R, et al. Newly diagnosed atrial fibrillation after acute ischemic stroke and transient ischemic attack: importance of immediate and prolonged continuous cardiac monitoring. J Stroke Cerebrovasc Dis. 2012 Apr;21(3):210-6.

6 Liao J, Khalid Z, Scallan C, Morillo C, O’Donnell M. Noninvasive cardiac monitoring for detecting paroxysmal atrial fibrillation or flutter after acute ischemic stroke: a systematic review. Stroke. 2007 Nov;38(11): 2935-40.

7 Lazzaro MA, Krishnan K, Prabhakaran S. Detection of atrial fibrillation with concurrent holter monitoring and continuous cardiac telemetry following ischemic stroke and transient ischemic attack. J Stroke Cerebrovasc Dis. 2012 Feb;21(2):89-93.

8 Grond M, Jauss M, Hamann G, Stark E, Veltkamp R, Nabavi D, et al. Improved detection of silent atrial fibrillation using 72-hour Holter ECG in patients with ischemic stroke: a prospective multicenter cohort study. Stroke. 2013 Dec;44(12):3357-64.

9 Thakkar S, Bagarhatta R. Detection of paroxysmal atrial fibrillation or flutter in patients with acute ischemic stroke or transient ischemic attack by Holter monitoring. Indian Heart J. 2014 Mar-Apr;66(2):188-92.

10 Gladstone DJ, Spring M, Dorian P, Panzov V, Thorpe KE, Hall J, et al.; EMBRACE Investigators and Coordinators. Atrial fibrillation in patients with cryptogenic stroke. N Engl J Med. 2014 Jun;370(26):2467-77.

11 Sanna T, Diener HC, Passman RS, Di Lazzaro V, Bernstein RA, Morillo CA, et al.; CRYSTAL AF Investigators. Cryptogenic stroke and underlying atrial fibrillation. $\mathrm{N}$ Engl $\mathrm{J}$ Med. 2014 Jun;370(26):2478-86.
12 Favilla CG, Ingala E, Jara J, Fessler E, Cucchiara B, Messé SR, et al. Predictors of finding occult atrial fibrillation after cryptogenic stroke. Stroke. 2015 May;46(5):1210-5.

13 Bhatt A, Majid A, Razak A, Kassab M, Hussain S, Safdar A. Predictors of occult paroxysmal atrial fibrillation in cryptogenic strokes detected by long-term noninvasive cardiac monitoring. Stroke Res Treat. 2011 Feb;2011: 172074.

14 Miller DJ, Khan MA, Schultz LR, Simpson JR, Katramados AM, Russman AN, et al. Outpatient cardiac telemetry detects a high rate of atrial fibrillation in cryptogenic stroke. J Neurol Sci. 2013 Jan;324(1-2):57-61.

15 Dussault C, Toeg H, Nathan M, Wang ZJ, Roux JF, Secemsky E. Electrocardiographic monitoring for detecting atrial fibrillation after ischemic stroke or transient ischemic attack: systematic review and meta-analysis. Circ Arrhythm Electrophysiol. 2015 Apr;8(2):263-9.

16 Sposato LA, Cipriano LE, Saposnik G, Ruíz Vargas E, Riccio PM, Hachinski V. Diagnosis of atrial fibrillation after stroke and transient ischaemic attack: a systematic review and meta-analysis. Lancet Neurol. 2015 Apr;14(4): 377-87.

17 Tayal AH, Tian M, Kelly KM, Jones SC, Wright DG, Singh D, et al. Atrial fibrillation detected by mobile cardiac outpatient telemetry in cryptogenic TIA or stroke. Neurology. 2008 Nov;71(21):1696-701.

18 Vingerhoets F, Bogousslavsky J, Regli F, Van Melle G. Atrial fibrillation after acute stroke. Stroke. 1993 Jan;24(1):26-30.

19 Doğan A, Tunç E, Oztürk M, Erdemoğlu AK. [Comparison of electrocardiographic abnormalities in patients with ischemic and hemorrhagic stroke]. Anadolu Kardiyol Derg. 2004 Jun;4(2):135-40.

20 van Bree MD, Roos YB, van der Bilt IA, Wilde AA, Sprengers ME, de Gans K, et al. Prevalence and characterization of ECG abnormalities after intracerebral hemorrhage. Neurocrit Care. 2010 Feb;12(1):50-5.

21 Maramattom BV, Manno EM, Fulgham JR, Jaffe AS, Wijdicks EF. Clinical importance of cardiac troponin release and cardiac abnormalities in patients with supratentorial cerebral hemorrhages. Mayo Clin Proc. 2006 Feb; 81(2):192-6.

22 Frontera JA, Parra A, Shimbo D, Fernandez A, Schmidt JM, Peter P, et al. Cardiac arrhythmias after subarachnoid hemorrhage: risk factors and impact on outcome. Cerebrovasc Dis. 2008;26(1):71-8.
23 Goldberger AL, Pavelec RS. Vagally-mediated atrial fibrillation in dogs: conversion with bretylium tosylate. Int J Cardiol. 1986 Oct; 13(1):47-55.

24 Huang CX, Zhao QY, Liang JJ, Chen H, Yang $B$, Jiang $H$, et al. Differential densities of muscarinic acetylcholine receptor and $\mathrm{I}(\mathrm{K}, \mathrm{ACh})$ in canine supraventricular tissues and the effect of amiodarone on cholinergic atrial fibrillation and $\mathrm{I}(\mathrm{K}, \mathrm{ACh})$. Cardiology. 2006; 106(1):36-43.

25 Sheng X, Scherlag BJ, Yu L, Li S, Ali R, Zhang $Y$, et al. Prevention and reversal of atrial fibrillation inducibility and autonomic remodeling by low-level vagosympathetic nerve stimulation. J Am Coll Cardiol. 2011 Feb;57(5):56371.

26 Yu L, Scherlag BJ, Li S, Sheng X, Lu Z, Nakagawa $\mathrm{H}$, et al. Low-level vagosympathetic nerve stimulation inhibits atrial fibrillation inducibility: direct evidence by neural recordings from intrinsic cardiac ganglia. J Cardiovasc Electrophysiol. 2011 Apr;22(4):455-63.

27 Ogawa M, Tan AY, Song J, Kobayashi K, Fishbein MC, Lin SF, et al. Cryoablation of stellate ganglia and atrial arrhythmia in ambulatory dogs with pacing-induced heart failure. Heart Rhythm. 2009 Dec;6(12):1772-9.

28 Zhao Q, Yu S, Huang H, Tang Y, Xiao J, Dai $Z$, et al. Effects of renal sympathetic denervation on the development of atrial fibrillation substrates in dogs with pacing-induced heart failure. Int J Cardiol. 2013 Sep;168(2):1672-3.

29 Sörös P, Hachinski V. Cardiovascular and neurological causes of sudden death after ischaemic stroke. Lancet Neurol. 2012 Feb; 11(2):179-88.

30 Billman GE. The LF/HF ratio does not accurately measure cardiac sympatho-vagal balance. Front Physiol. 2013 Feb;4:26.

31 Chidambaram H, Gnanamoorthy K, Suthakaran PK, Rajendran K, Pavadai C. Assessment of Autonomic Dysfunction in Acute Stroke Patients at a Tertiary Care Hospital. J Clin Diagn Res. 2017 Feb;11(2):OC28-31.

32 Robinson TG, Dawson SL, Eames PJ, Panerai RB, Potter JF. Cardiac baroreceptor sensitivity predicts long-term outcome after acute ischemic stroke. Stroke. 2003 Mar;34(3):70512.

33 Tang SC, Jen HI, Lin YH, Hung CS, Jou WJ, Huang PW, et al. Complexity of heart rate variability predicts outcome in intensive care unit admitted patients with acute stroke. J Neurol Neurosurg Psychiatry. 2015 Jan;86(1): 95-100. 
34 Ruthirago D, Julayanont P, Tantrachoti P, Kim J, Nugent K. Cardiac Arrhythmias and Abnormal Electrocardiograms After Acute Stroke. Am J Med Sci. 2016 Jan;351(1):112-8.

35 Mai LM, Sposato LA. Insular damage, death and newly diagnosed atrial fibrillation in intracerebral hemorrhage: stroke-induced heart injury as the potential missing link. Eur J Neurol. 2018 Mar;25(3):423-4.

36 Zamrini EY, Meador KJ, Loring DW, Nichols FT, Lee GP, Figueroa RE, et al. Unilateral cerebral inactivation produces differential left/ right heart rate responses. Neurology. 1990 Sep;40(9):1408-11.

37 Colivicchi F, Bassi A, Santini M, Caltagirone C. Prognostic implications of right-sided insular damage, cardiac autonomic derangement, and arrhythmias after acute ischemic stroke. Stroke. 2005 Aug;36(8):1710-5.

38 Colivicchi F, Bassi A, Santini M, Caltagirone C. Cardiac autonomic derangement and arrhythmias in right-sided stroke with insular involvement. Stroke. 2004 Sep;35(9):2094-8.

39 Meyer S, Strittmatter M, Fischer C, Georg T, Schmitz B. Lateralization in autonomic dysfunction in ischemic stroke involving the insular cortex. Neuroreport. 2004 Feb;15(2):357-61.

40 Manea MM, Comsa M, Minca A, Dragos D, Popa C. Brain-heart axis-review Article. J Med Life. 2015 Jul-Sep;8(3):266-71.

41 Di Angelantonio E, Fiorelli M, Toni D, Sacchetti ML, Lorenzano S, Falcou A, et al. Prognostic significance of admission levels of troponin I in patients with acute ischaemic stroke. J Neurol Neurosurg Psychiatry. 2005 Jan;76(1):76-81.

42 Bugnicourt JM, Rogez V, Guillaumont MP, Rogez JC, Canaple S, Godefroy O. Troponin levels help predict new-onset atrial fibrillation in ischaemic stroke patients: a retrospective study. Eur Neurol. 2010;63(1):24-8.

43 Saini HK, Tripathi ON, Zhang S, Elimban V, Dhalla NS. Involvement of $\mathrm{Na}+/ \mathrm{Ca} 2+\mathrm{ex}-$ changer in catecholamine-induced increase in intracellular calcium in cardiomyocytes. Am J Physiol Heart Circ Physiol. 2006 Jan; 290(1):H373-80

44 Samuels MA. Neurogenic heart disease: a unifying hypothesis. Am J Cardiol. 1987 Dec; 60(18):15J-9J.

45 Gauberti M, Montagne A, Quenault A, Vivien D. Molecular magnetic resonance imaging of brain-immune interactions. Front Cell Neurosci. 2014 Nov;8:389.

46 Doll DN, Barr TL, Simpkins JW. Cytokines: their role in stroke and potential use as biomarkers and therapeutic targets. Aging Dis. 2014 Oct;5(5):294-306

47 Fumagalli S, Perego C, Pischiutta F, Zanier ER, De Simoni MG. The ischemic environment drives microglia and macrophage function. Front Neurol. 2015 Apr;6:81.

48 Shichita T, Hasegawa E, Kimura A, Morita R, Sakaguchi R, Takada I, et al. Peroxiredoxin family proteins are key initiators of post-ischemic inflammation in the brain. Nat Med. 2012 Jun;18(6):911-7.
49 Liesz A, Dalpke A, Mracsko E, Antoine DJ, Roth S, Zhou W, et al. DAMP signaling is a key pathway inducing immune modulation after brain injury. J Neurosci. 2015 Jan;35(2): 583-98.

50 Xiong XY, Liu L, Yang QW. Functions and mechanisms of microglia/macrophages in neuroinflammation and neurogenesis after stroke. Prog Neurobiol. 2016 Jul;142:23-44.

51 Becker K. Autoimmune responses to brain following stroke. Transl Stroke Res. 2012 Sep; 3(3):310-7.

52 Becker KJ, Kindrick DL, Lester MP, Shea C, Ye ZC. Sensitization to brain antigens after stroke is augmented by lipopolysaccharide. J Cereb Blood Flow Metab. 2005 Dec;25(12): 1634-44.

53 Jauch EC, Lindsell C, Broderick J, Fagan SC, Tilley BC, Levine SR; NINDS rt-PA Stroke Study Group. Association of serial biochemical markers with acute ischemic stroke: the National Institute of Neurological Disorders and Stroke recombinant tissue plasminogen activator Stroke Study. Stroke. 2006 Oct; 37(10):2508-13.

54 van der Bilt IA, Vendeville JP, van de Hoef TP, Begieneman MP, Lagrand WK, Kros JM, et al. Myocarditis in patients with subarachnoid hemorrhage: A histopathologic study. J Crit Care. 2016 Apr;32:196-200.

$55 \mathrm{Hu}$ YF, Chen YJ, Lin YJ, Chen SA. Inflammation and the pathogenesis of atrial fibrillation. Nat Rev Cardiol. 2015 Apr;12(4):230-43.

$56 \mathrm{He} \mathrm{B}, \mathrm{Lu}$ Z, He W, Huang B, Jiang H. Lowintensity atrial ganglionated plexi stimulation decreases the serum level of inflammatory factors in canine. Heart Lung Circ. 2015 Apr; 24(4):407-10.

57 Zhao Q, Zhang S, Huang H, Zhang Y, Deng $\mathrm{H}$, Yu S, et al. Inflammation abnormalities and inducibility of atrial fibrillation after epicardial ganglionated plexi ablation. Arch Cardiovasc Dis. 2011 Apr;104(4):227-33.

58 Stavrakis S, Humphrey MB, Scherlag BJ, Hu Y, Jackman WM, Nakagawa H, et al. Low-level transcutaneous electrical vagus nerve stimulation suppresses atrial fibrillation. J Am Coll Cardiol. 2015 Mar;65(9):867-75.

59 Wakili R, Voigt N, Kääb S, Dobrev D, Nattel $\mathrm{S}$. Recent advances in the molecular pathophysiology of atrial fibrillation. J Clin Invest. 2011 Aug;121(8):2955-68.

60 Saba S, Janczewski AM, Baker LC, Shusterman V, Gursoy EC, Feldman AM, et al. Atrial contractile dysfunction, fibrosis, and arrhythmias in a mouse model of cardiomyopathy secondary to cardiac-specific overexpression of tumor necrosis factor-\{alpha\}. Am J Physiol Heart Circ Physiol. 2005 Oct;289(4):H1456-67.

61 Liew R, Khairunnisa K, Gu Y, Tee N, Yin NO, Naylynn TM, et al. Role of tumor necrosis factor- $\alpha$ in the pathogenesis of atrial fibrosis and development of an arrhythmogenic substrate. Circ J. 2013;77(5):1171-9.
62 Nishimoto N, Kishimoto T. Interleukin 6 from bench to bedside. Nat Clin Pract Rheumatol. 2006 Nov;2(11):619-26.

63 Liao $\mathrm{CH}$, Akazawa $\mathrm{H}$, Tamagawa M, Ito K, Yasuda N, Kudo Y, et al. Cardiac mast cells cause atrial fibrillation through PDGF-Amediated fibrosis in pressure-overloaded mouse hearts. J Clin Invest. 2010 Jan;120(1): 242-53.

64 Musa H, Kaur K, O’Connell R, Klos M, Guerrero-Serna G, Avula UM, et al. Inhibition of platelet-derived growth factor- $\mathrm{AB}$ signaling prevents electromechanical remodeling of adult atrial myocytes that contact myofibroblasts. Heart Rhythm. 2013 Jul;10(7):104451.

65 Helbing T, Olivier C, Bode C, Moser M, Diehl P. Role of microparticles in endothelial dysfunction and arterial hypertension. World J Cardiol. 2014 Nov;6(11):1135-9.

66 Lackner P, Dietmann A, Beer R, Fischer M, Broessner G, Helbok R, et al. Cellular microparticles as a marker for cerebral vasospasm in spontaneous subarachnoid hemorrhage. Stroke. 2010 Oct;41(10):2353-7.

$67 \mathrm{Li} \mathrm{P}$, Qin C. Elevated circulating VEcadherin+CD144+endothelial microparticles in ischemic cerebrovascular disease. Thromb Res. 2015 Feb;135(2):375-81.

68 Huang M, Hu YY, Dong XQ. High concentrations of procoagulant microparticles in the cerebrospinal fluid and peripheral blood of patients with acute basal ganglia hemorrhage are associated with poor outcome. Surg Neurol. 2009 Nov;72(5):481-9.

69 Wang $\mathrm{H}$, Yan HM, Tang MX, Wang ZH, Zhong M, Zhang Y, et al. Increased serum levels of microvesicles in nonvalvular atrial fibrillation determinated by ELISA using a specific monoclonal antibody AD-1. Clin Chim Acta. 2010 Nov;411(21-22):1700-4.

70 van den Berg NW, Kawasaki M, Berger WR Neefs J, Meulendijks E, Tijsen AJ, et al. MicroRNAs in Atrial Fibrillation: from Expression Signatures to Functional Implications. Cardiovasc Drugs Ther. 2017 Jun;31(3):34565.

71 Tan KS, Armugam A, Sepramaniam S, Lim KY, Setyowati KD, Wang CW, et al. Expression profile of MicroRNAs in young stroke patients. PLoS One. 2009 Nov; 4(11):e7689.

72 Long G, Wang F, Li H, Yin Z, Sandip C, Lou $\mathrm{Y}$, et al. Circulating miR-30a, miR-126 and let-7b as biomarker for ischemic stroke in humans. BMC Neurol. 2013 Nov;13(1):178.

73 Wei XJ, Han M, Yang FY, Wei GC, Liang ZG, Yao H, et al. Biological significance of miR126 expression in atrial fibrillation and heart failure. Braz J Med Biol Res. 2015 Nov;48(11): 983-9.

74 Sposato LA, Cerasuolo JO, Cipriano LE, Fang J, Fridman S, Paquet M, et al.; PARADISE Study Group. Atrial fibrillation detected after stroke is related to a low risk of ischemic stroke recurrence. Neurology. 2018 Mar; 90(11):e924-31. 